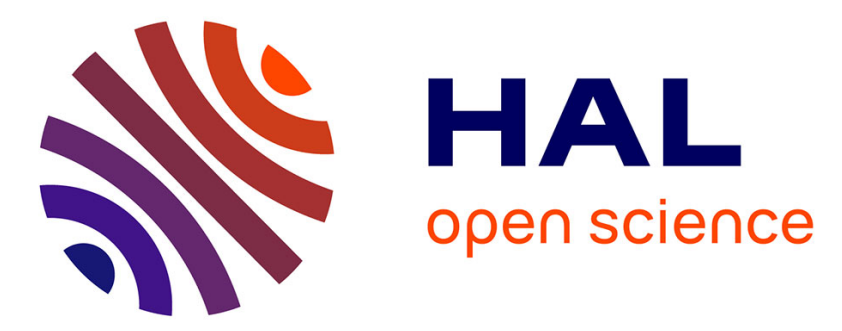

\title{
Strategic territorial deployment of hospital pharmacy robots using a stochastic p-robust optimization approach
}

Carlos Franco, Vincent Augusto, Thierry Garaix, Edgar Alfonso-Lizarazo, Magali Bourdelin, Hervé Bontemps

\section{- To cite this version:}

Carlos Franco, Vincent Augusto, Thierry Garaix, Edgar Alfonso-Lizarazo, Magali Bourdelin, et al.. Strategic territorial deployment of hospital pharmacy robots using a stochastic p-robust optimization approach. 2018 IEEE 14th International Conference on Automation Science and Engineering (CASE), Aug 2018, Munich, France. pp.390-395, 10.1109/COASE.2018.8560374 . hal-02024966

\section{HAL Id: hal-02024966 https://hal.science/hal-02024966}

Submitted on 4 Mar 2019

HAL is a multi-disciplinary open access archive for the deposit and dissemination of scientific research documents, whether they are published or not. The documents may come from teaching and research institutions in France or abroad, or from public or private research centers.
L'archive ouverte pluridisciplinaire HAL, est destinée au dépôt et à la diffusion de documents scientifiques de niveau recherche, publiés ou non, émanant des établissements d'enseignement et de recherche français ou étrangers, des laboratoires publics ou privés. 


\title{
Strategic territorial deployment of hospital pharmacy robots using a stochastic p-robust optimization approach
}

\author{
Carlos Franco, Vincent Augusto, Thierry Garaix, Edgar Alfonso-Lizarazo, Magali Bourdelin, Hervé \\ Bontemps
}

\begin{abstract}
Automation in healthcare is a major challenge to improve quality of service while compressing costs. In particular, correct administration of medicines to patients is crucial to ensure quality of care during hospitalization and minimize medication errors. Mistakes are more likely to happen when medicine administration is done manually (dispensing, ordering or administrating). To reduce the risks related to medication errors, automation of the pharmacy processes appears as an appropriately tool to solve this situation. In this paper, we have proposed a new mathematical model to optimize the processes related to unit-doses management and prescriptions preparation in a network of hospitals. To model the uncertainty associated with the demand of medicines, the concept of p-robustness is included; the concept of resilience is also considered to model the risk of centralized distribution processes.
\end{abstract}

\section{INTRODUCTION}

Access to medicines and reliability in the pharmaceutical requirements of patients is one of the most important challenge in primary healthcare. To deal with this, automation technologies have become a support in the pharmaceutical services that helps hospitals to minimize human errors, minimize costs and improve efficiency of processes. In this way, the term pharmacy automation appears as the process of automating the routine tasks performed in pharmacies [1][2]. The related work within pharmacies consists in storing medicines, making unit-doses, preparing prescriptions and delivering prescriptions to patients. Critical errors may occur during the two last steps. Automatic dispensing systems is an innovative tool that allows hospitals not only to satisfy requirements of patients but also optimize the distribution of medicines across a network of interconnected facilities [3].

In this paper, we present a real application of an optimization of the automation process within a network of hospitals considering specific characteristics of the storage, packaging and distribution of medicines processes. Such problem can be addressed as a location-allocation problem. We have developed a mixed integer linear program (MILP) adding a special feature to take into account risks in the pharmaceutical supply chains such as resilience. Also, we extended the proposed MILP to include uncertainty in the demand since satisfaction of medicines requirements within

C. Franco is with Universidad del Rosario, Bogotá, Colombia, Calle 200 entre Autopista Norte y Carrera séptima and also with Universidad de la Sabana, Chia, Colombia (corresponding author, phone: 05712970200 ext: 3942; e-mail: carlosa.franco@urosario.edu.co and carlosfrfr@unisabana.edu.co).

V. Augusto and T. Garaix are with Mines Saint-Etienne, Univ. Clermont Auvergne, CNRS, UMR 6158 LIMOS, Center for Health Care Engineering, F-42023, Saint-Etienne, France (e-mail: [augusto;garaix]@emse.fr). hospitals is a crucial objective regarding the quality of care. We use the concept of p-robustness to model the uncertainty on the demand and analyzed the results obtained on a real case study. We do not consider uncertainty from the production side (e.g. routing time uncertainty or probability of machine failure) since it was considered less important by health practitioners; however, such elements will be considered in future works.

This paper is organized as follows. A literature review on location and location-allocation is provided in Section II. The proposed mathematical model and its extensions are detailed in Section III. The results obtained with each model over an application to a real case study are provided in Section IV. Finally, conclusions are developed in Section V.

\section{LITERATURE REVIEW}

Location-allocation problems solution approaches and applications have been widely studied in the scientific literature [4][5][6]. We consider two types of contributions: (i) applications of location and location-allocation problems in the healthcare context, and (ii) theoretical developments and applications in other domains.

An application of location and reorganization in health systems is developed in [7]. This paper presents a real application in a healthcare system in Italy. Two problems are considered: (i) healthcare reorganization problem considering regional guidelines that aim to replace some ordinary admissions by ambulatory or home cares and (ii) build a new model that considers the demand satisfaction and the increasing of the hospital capacity. A similar work is proposed in [8].

A location-allocation model under uncertainty for hospital network planning is proposed in [9]. The total cost of the network of hospitals is minimized as the expected distance to facilities. The authors developed a two-stage model: the first stage is limited to decisions related to location and the second stage involves location and allocation decisions. This model is applied to a real case in Portugal with two types of hospitals: non-specialized hospitals close to the population and high specialized ones that could stand far from the demand.

E. Alfonso-Lizarazo is with University of Lyon, University Jean MonnetSt-Etienne, LASPI, EA3059, F-42023, Saint-Etienne, France .(e-mail: edgar.alfonso.lizarazo@univ-st-etienne.fr)

M. Bourdelin and H. Bontemps are with Hôpital Nord Ouest, BP 80436, 69655 Villefranche-sur-Saône, France (e-mail: [mbourdeling;

hbontemps]@lhopitalnordouest.fr). 
A similar two-stage approach is developed in [10]. The main idea is to determine the hospital location, then services and machines allocation and finally assign patients to services. The authors proposed a bi-objective robust model assuming that costs are uncertain. Other multi-objective models are applied to the location and sizing of medical departments in a hospital network in [11] and [12]. A similar problem is studied in [13] for emergency medical service stations.

A robust approximation for a multi-period locationallocation problem of pharmaceutical centers is proposed in [14]. A multi-objective model is used by considering the minimization of costs and the maximization of customer's satisfaction. Epsilon constraint approach is used for solving the bi-objective model. The robustness is considered regarding the uncertainty in demand.

A healthcare application applied to emergency response in case of disaster is developed in [15]. A hybrid model is presented combining a simulation model and a nonlinear optimization model of the assignment of workers to workstations which is solved by simulation and a heuristic optimization algorithm. Decisions are related to the locations for dispensing aids and the design of the logistics to supply the demand. A review for location problems in emergencies is presented in [16].

In [17] is proposed a model to locate services in a musculoskeletal physiotherapy department in which the patient behavior is modelled using heuristics. The model considers the individuality of patients along with the availability of resources. It uses simulation where scenarios correspond to different configurations of services. Several applications of facility location and extension are presented in these two surveys [18], [19],. Selecting locations for waste material in healthcare in [20] is another relevant application of location problems in healthcare.

There are some recent developments in location problems. A combined problem of location and routing problem is defined in [21] and solved with a genetic algorithm. Approximate dynamic programming is used for solving a stochastic location problem in [22]. The problem consists in determine the optimal policy that indicates the sources and destinations of transshipments where the demand is stochastic. Other applications for different variants of location-allocation problems can be found in [23]-[26].

Regarding the literature, our paper makes the following contributions: (i) model a real case application of optimizing the automation applied in a healthcare network, (ii) use the logistic concept of resilience to model the risk of failure in robots in a very high and sensitive distribution network and (iii) model uncertainty using the concept of p-robustness.

\section{FORMAL MODEL}

\section{A. Problem Description}

The optimization problem considered in this paper is modeled as an extension of a location-allocation routing problem. The objective function consists in determining the best location and allocation of different types of robots for a network of hospitals. Three types of robots are considered: (i) robots that process medicines from suppliers and produce unit- doses (i.e. medicines are extracted from their boxes and cut into unit-doses) named cutting machines; (ii) robots that store unit-doses named storage machines, and (iii) robots that process unit-doses to compose personalized prescriptions depending on patient requirements (i.e. unit-doses of different medicines are grouped together in a plastic bag to compose a prescription) named prescription machines. Storage and prescription machines can be bought with different settings, i.e. different costs and capacities. Each hospital of the network can have robots of each type.

For example, let us consider a network of three hospitals $\mathrm{H} 1, \mathrm{H} 2$ and $\mathrm{H} 3$. The network design is: $\mathrm{H} 1$ has one cutting machine producing unit-doses $(\mathrm{R} 1)$, one prescription machine (R2) and one storage machine (R4); H2 has one prescription machine (R3) and one storage machine (R5); H3 has no robot. The transportation plan is: H1's robot R1 produces unit-doses for H1 (stored in R4) and H2 (stored in R5); H1's robot R2 produces prescriptions for $\mathrm{H} 1$ only. Unit-doses are transported from $\mathrm{H} 1$ to $\mathrm{H} 2$. H2's robot $\mathrm{R} 3$ produces prescriptions using unit-doses received from $\mathrm{H} 1$ for $\mathrm{H} 2$ and $\mathrm{H} 3$. Prescriptions are transported from $\mathrm{H} 2$ to $\mathrm{H} 3$. Fig. 1 illustrates this process: dotted line arrows model unit-doses flows whereas hard line arrows model prescription flows.

Figure 1. Ilustrative example

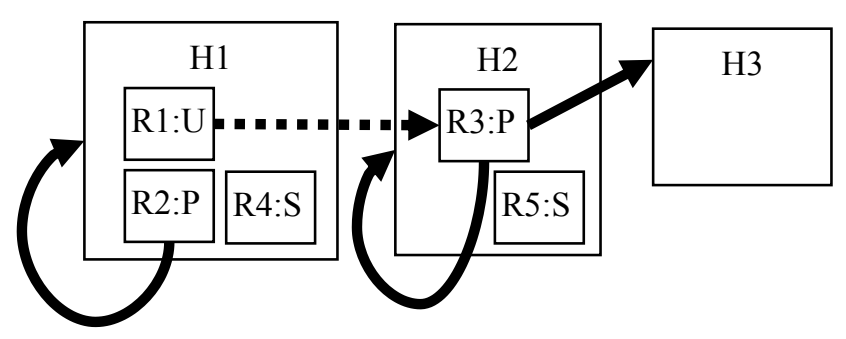

Two types of decisions are considered: (i) the number of robots of each type in each hospital, and (ii) the distribution flow over the network (i.e. which hospitals provide unit-doses and/or prescriptions for which hospital). Two mathematical models are proposed. In the first one the demand for medicines for each hospital is deterministic whereas in the second model this demand is stochastic.

\section{B. Deterministic Model}

In order to define the problem described above as a MILP, we introduce the following sets, parameters and variables:

Sets:

- $\quad H$ : set of hospitals

- $\quad P M$ : set of settings of prescription machines

- $\quad S M$ : set of settings of storage machines

Parameters:

- UR: utilization rate of machines

- $T C U_{i j}$ : transportation costs of unit-doses between hospitals

- $T C P_{i j}$ : transportation costs of prescriptions between hospitals

- $O P_{i}$ : annual opening hours per hospital

- $D_{i}$ : requirements of unit-doses per year in each hospital 
- $\quad P U$ : price of unit-doses (cutting)

- $D H_{i}$ : number of daily working hours per hospital

- $\quad \operatorname{CapP} P_{p m}$ : capacities of preparing prescriptions per setting

- $\operatorname{Cost} P P_{p m}$ : cost of prescription machines for each setting

- $A m$ : years of investment

- $U D P_{i}$ : number of unit-doses per prescription in each hospital

- $\quad \operatorname{CapS}_{\text {sm }}$ : storage capacity of each setting

- $\operatorname{Cost} S_{\mathrm{sm}}$ : cost of storage machines for each setting

- $\operatorname{CostC}$ : unitary cost of cutting machines

- CapProd: capacity of cutting machines

- $M$ : big number

Decision variables:

- $A_{i j}$ : number of unit-doses distributed each year between hospitals

- $\quad B_{i j}$ : number of prescriptions distributed each year between hospitals

- $N_{i}$ : number of cutting machines in each hospital

- $X_{i j}=1$ if there is transportation of unit-doses between hospitals, 0 otherwise

- $Y_{i j}=1$ if there is transportation of prescriptions between hospitals, 0 otherwise

- $\quad P M_{p m i}$ : number of dispatching machines per type in each hospital

- $S M_{s m i}$ : number of storage machines per type in each hospital

The mathematical model is described as follows:

$\operatorname{Min} z=P U * A m * \sum_{i \in H} \sum_{j \in H} A_{i j}+$

$\sum_{i \in H} \sum_{j \in H}\left(T C U_{i j} * A m * X_{i j}+Y_{i j} * A m * 2 * T C P_{i j}\right)+$

$\sum_{p m \in P M} \sum_{i \in H} \operatorname{CostPP}_{p m} * P M_{p m i}+$

$\sum_{s m \in S M} \sum_{i \in H} \operatorname{Cost}_{s m} * S M_{s m i}+\sum_{i \in H} \operatorname{CostC} * N_{i}$

subject to:

$N_{i} *$ CapProd $* O P_{i} * U R \geq \sum_{k \in H} A_{i k} \forall i \in H$

$\sum_{p m \in P M} \operatorname{CapP} P_{p m} * P M_{p m i} * O P_{i} * U R \geq$

$\sum_{k \in H} B_{i k} * U D P_{k} \quad \forall i H$

$\sum_{s m \in S M} \operatorname{CapS}_{s m} * S M_{s m i} \geq \sum_{j \in H} A_{i j} * \frac{5}{365} \forall i \in H$

$\sum_{j \in H} A_{j i}=\sum_{j \in H} B_{i j} * U D P_{j} \quad \forall i \in H$

$\sum_{p m \in P M} P M_{p m i} \leq \sum_{s m \in S M} S M_{s m i} \forall i \in H$

$\sum_{i \in H} U D P_{j} * B_{i j} \geq D_{j} \quad \forall j \in H$

$A_{i j} \leq M * X_{i j} \quad \forall i, j \in H$

$B_{i j} \leq M * Y_{i j} \quad \forall i, j \in H$.

$$
\begin{aligned}
& X_{j i} \leq 1-X_{i j} \quad \forall i, j \in H \mid i<>j \\
& \sum_{i \in H} X_{i j} \leq 1 \quad \forall j \in H \\
& \sum_{i \in H \mid i<>j} Y_{i j} \leq 1 \quad \forall j \in H \\
& \text { CapProd } * N_{i} * D H_{i} * U R \geq \frac{1}{365} \quad \forall i \in H \\
& \sum_{i \in H \mid i=j} Y_{i j} \leq \sum_{p m \in P M} M * P M_{p m j} \quad \forall j \in H \\
& C a p P r o d * N_{i} \geq \sum_{j \in H} A_{i j} \quad \forall i H \\
& \sum_{p m \in P M} P M_{p m i} \geq \sum_{j \in H} B_{i j} \quad \forall i \in H \\
& \sum_{p m \in P M} C_{p P P P_{p m}} * P M_{p m j} * D H_{i} * U R \geq \sum_{j \in H} B_{i j} * \\
& \frac{4}{365} \quad \forall i \in H \\
& A_{h j}, B_{h j}, N_{h}, P M_{p m h}, S M_{s m h}>=0 ; X_{h j}, Y_{h j} \in\{0,1\}
\end{aligned}
$$

The mathematical model uses 3 sets: (i) set of hospitals $H$ that indicates the hospitals in the network, (ii) set of possible settings of prescription machines $P M$, and (iii) the set of possible settings for storage machines $S M$. Objective function (1) minimizes the overall costs composed by (i) cutting costs, (ii) transportation costs and (iii) investment costs. Cutting costs represents the cost of producing unit-doses. Transportation costs are divided into transportation of unitdoses and transportation of prescriptions. The investment cost is divided into costs of prescription/storage machines and cost of unit-doses machines. Equation (2) guarantees that cutting machines are enough to satisfy the distribution of unit-doses between hospitals. Equation (3) ensures that machines for preparing prescriptions in each hospital are enough to satisfy the prescriptions distributed between hospitals (the capacity of prescriptions for each type of machine is expressed in terms of unit-doses). Equation (4) models the storage capacity. Such capacity for each hospital must guarantee at least five days of distribution of unit-doses. Prescriptions contain a certain number of unit-doses, equation (5) is developed to balance the number of unit-doses received and the number of unit-doses dispatched in terms of prescriptions for each hospital. Equation (6) determines that for each hospital there must be at maximum the same number of prescription machines and storage machines. Satisfaction of demand in terms of unitdoses is modeled in (7). Equations (8) and (9) ensure the relationship between the binary variables of distribution of unit-doses and prescriptions with the integer variables of distribution of unit-doses and prescriptions. Equation (10) ensures that distribution of unit-doses is not allowed in two directions, this means that a hospital can supply other hospital, but the supplied hospital cannot supply in return the previous one. For medicines flow requirements, each hospital can only receive unit-doses from only one hospital (11). Equation (12) models that each hospital can only receive prescriptions for at most one hospital or by itself. Equation (13) guarantees that capacity of unit-doses machines in terms of unit-doses is enough for having daily capacity of 
distribution of unit-doses. Equation (14) ensures that each hospital only can satisfy its own demand if prescription machines are implanted there. Distribution of unit-doses can only be made by hospitals that locate unit-doses machines in their own (15). Distribution of prescriptions can only be made by hospitals that locate prescriptions machines in their own hospitals (16). Equation (17) ensures that in each hospital that will distribute prescriptions there has to be enough daily capacity to ensure at least 4 days of making prescriptions. Finally equation (18) ensures the domain definition of variables.

\section{Resilience and robustness in pharmaceutical supply chains}

We have used the concept of risk and resilience in supply chains proposed in [27][28] as the ability of organizations to bounce back from large scale disruptions such as random events, accidents, negligence, intentional disruptions, natural disasters, among others [29]. In this way and as the distribution of medicines is a very important issue where backorders or stockouts are not allowed because of patient's health, we introduce additional constraints to ensure that locations of robots are not centralized in one hospital, increasing the flexibility in the distribution of medicines. Since decisions of purchasing robots are made on a strategic level, we have considered the uncertainty in demand as a factor of analysis. For that we have added and modified the following elements using the concept of p-robustness developed in [30]. To describe the model we have used the strategy presented in [9] which formulates the mathematical model as non-scenario dependent. The new terms added to the mathematical model are as follows:

Sets:

- $\quad S C N$ : set of scenarios of variation of demand

Parameters:

- $D_{s c i}:$ requirements of unit-doses by hospital in each scenario

- $q_{s c}$ : probability that a scenario occurs

- $\rho$ : desired robustness level

The variation of the mathematical model is as follows:

$=\sum_{s c n \in S C N}^{\text {Min } z} q_{s c n}$
$*\left[\begin{array}{l}\sum_{i \in H} \sum_{j \in H} P U * A m * A_{i j}+\sum_{i \in H} \sum_{j \in H}\left(T C U_{i j} * A m * X_{i j}+Y_{i j} * A m * 2 * T C P_{i j}\right) \\ +\sum_{p m \in P M} \sum_{i \in H} \operatorname{CostP} P_{p m} * P M_{p m i}+\sum_{s m \in S M} \sum_{i \in H} \operatorname{Cost} S_{s m} * S M_{s m i}+\sum_{i \in H} \operatorname{CostC} * N_{i}\end{array}\right]$

$\sum_{i \in H} \sum_{j \in H} P U * A m * A_{i j}+\sum_{i \in H} \sum_{j \in H}\left(T C U_{i j} * A m * X_{i j}+\right.$

$\left.Y_{i j} * A m * 2 * T C P_{i j}\right)+\sum_{p m \in P M} \sum_{i \in H} \operatorname{CostP} P_{p m} * P M_{p m i}+$

$\sum_{s m \in S M} \sum_{i \in H} \operatorname{CostS}_{s m} * S M_{s m i}+\sum_{i \in H} \operatorname{CostC} * N_{i} \leq(1+$

$\rho)) * Z^{*}{ }_{s c n}$

$\sum_{i \in H} U D P_{j} * B_{i j} \geq D_{s c j} \quad \forall j \in H, \forall S C \in S C$
Constraints (2) - (18) are similar except for variables having the index of scenarios of demand (Equation (7) is replaced by Equation (21)). The new objective function (19) minimizes the expected transportation cost and the location of machines purchased overall scenarios. In (20) is enforced the probustness condition that is a measure that combines the minimization of the expected costs and the minimization of the worst-case cost or regret.

\section{NUMERICAL RESULTS}

A real case study related to the "Rhône Nord Beaujolais Dombes" territorial hospital network is proposed to illustrate the results of the mathematical model and its applicability in a realistic case. The network located in the north of Lyon (France) has 8 different hospitals spread in the region, and each one of them has different consumptions and different types of illnesses treated; also because of the size, they have variations in demand between them. For the robust version we have generated 30 different scenarios of demand, in which the variations were generated with the average value defined by the hospitals network with a range of (+/-) $30 \%$.

Results are analyzed considering performance (computational times and complexity of the models), key performance indicators (costs of the provided solutions) and qualitative indicators (network design).

The example provided is analyzed considering the whole hospitals network and with full opening hours. The instance used is defined as follows:

- 8 hospitals in the network;

- Overture times (annual) varies between 1,690 and 2,820 hours per year;

- Demand of prescriptions varies between 387,581 and 3,038,490 per year;

- The supplier has one setting of machines for each type of process (cutting and packaging for cutting machines, storage and preparation of prescriptions for prescription machines);

- Daily opening hours varies between $6.5 \mathrm{~h}$ to $8.5 \mathrm{~h}$;

- Capacities of machines can have a small extension instead of buying another machine of the same type.

Table I provides statistics related to the deterministic model and the p-robust version. Complexity of the p-robust model is not high compared to the deterministic model whereas computational times are doubled. However, total CPU time remains very low considering the strategic nature of the problem. 
TABLE I. COMPUTATIONAL RESULTS

\begin{tabular}{|l|c|c|c|}
\hline \multirow{2}{*}{ Problem } & \multicolumn{3}{|c|}{ Computational results } \\
\cline { 2 - 4 } & No. of variables & $\begin{array}{c}\text { No. } \text { of } \\
\text { constraints }\end{array}$ & CPU time (s) \\
\hline Deterministic & 328 & 304 & 26.8 \\
\hline P-robust & 328 & 334 & 54.6 \\
\hline
\end{tabular}

As a qualitative analysis, the network design of the deterministic model and the robust version is presented in Figure 2. The triangles represent the 8 hospitals in the network. Dotted lines represent distribution of unit-doses and continuous lines represent distribution of prescriptions in both models. In the deterministic result, it can be observed that the network design is very centralized, each type of robots is allocated in hospital 4 and from that hospital are distributed prescriptions for the rest of hospitals in the network. In the robust version, the network design is distributed into three hospitals, hospitals 1, 7 and 4. In each hospital is allocated robots to prepare and storage unit-doses and prepare prescriptions, also each hospital where are located robots satisfy its own demand and the demand of hospitals assigned.

Figure 1. Results of the configuration of the network

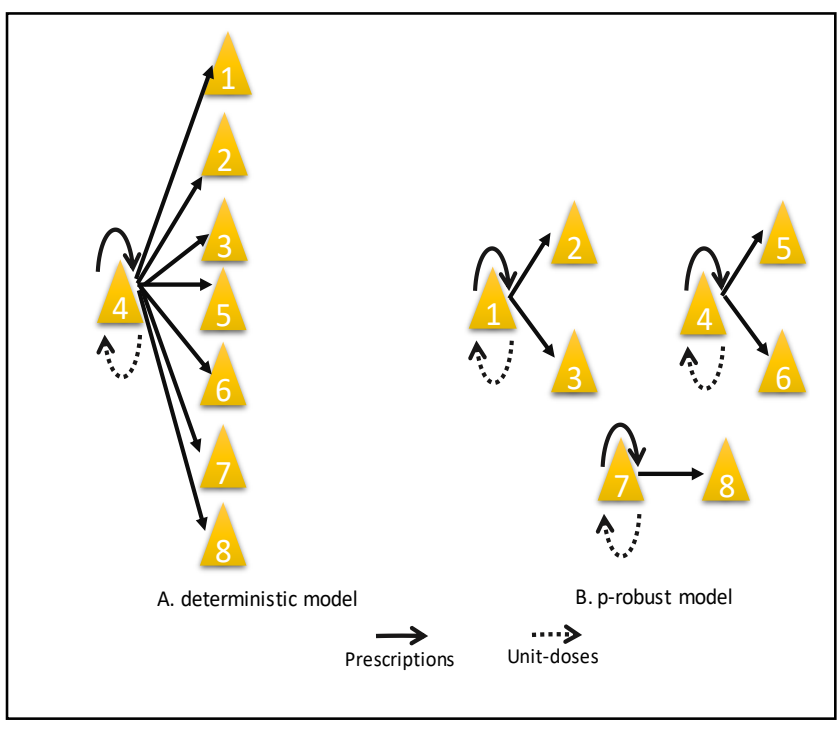

Tables II, III and IV provide results of the network design of both models with their capacities. In Table II results of the deterministic version including number of robots located and their capacities in units per week are presented. Tables III and IV present the network design of the p-robust model. Table III present the configuration of the number of robots in each hospital and Table IV present the capacities in each hospital.

TABLE II. DETERMINISTIC CONFIGURATION

\begin{tabular}{|l|c|c|}
\hline \multirow{2}{*}{\multicolumn{1}{|c|}{ Type of robots }} & \multicolumn{2}{c|}{ Configuration and capacities } \\
\cline { 2 - 3 } & \#of robots & Capacity (units per week) \\
\hline Preparing unit-doses & 5 & 200,000 \\
\hline Storage & 3 & 135,000 \\
\hline Preparing Prescriptions & 3 & 120,000 \\
\hline
\end{tabular}

TABLE III. P-ROBUST CONFIGURATION

\begin{tabular}{|l|c|c|c|}
\hline \multirow{2}{*}{$\begin{array}{c}\text { Type of } \\
\text { robots }\end{array}$} & \multicolumn{3}{|c|}{ Number of robots } \\
\cline { 2 - 4 } $\begin{array}{l}\text { Preparing } \\
\text { unit-doses }\end{array}$ & 1 & $\boldsymbol{H} 4$ & $\boldsymbol{H} 7$ \\
\hline Storage & 1 & 5 & 1 \\
\hline $\begin{array}{l}\text { Preparing } \\
\text { Prescriptions }\end{array}$ & 1 & 3 & 1 \\
\hline
\end{tabular}

TABLE IV. P-ROBUST CAPACITIES

\begin{tabular}{|l|c|c|c|}
\hline \multirow{2}{*}{$\begin{array}{c}\text { Type of } \\
\text { robots }\end{array}$} & \multicolumn{3}{|c|}{ Capacities in units per week } \\
\cline { 2 - 4 } & $\boldsymbol{H 1}$ & $\boldsymbol{H} 4$ & $\boldsymbol{H} 7$ \\
\hline $\begin{array}{l}\text { Preparing } \\
\text { unit-doses }\end{array}$ & 40,000 & 250,000 & 45,000 \\
\hline Storage & 45,000 & 135,000 & 45,000 \\
\hline $\begin{array}{l}\text { Preparing } \\
\text { Prescriptions }\end{array}$ & 40,000 & 12,000 & 40,000 \\
\hline
\end{tabular}

Several conclusions can be formulated regarding these results:

- Using the concept of resilience in very sensitive distribution networks as healthcare distribution of medicines allows the p-robust version to obtain a desirable locationallocation results in which the hospitals are not dependent of only one hospital in which any disruption can be translate into the not satisfaction of the patient's demand.

- The p-robust version model allows hospitals to satisfy the demand including its variation while the first model cannot satisfy the demand out of the deterministic range.

\section{CONCLUSION}

The objective of this paper is to develop a real case application of optimization in automating the process of preparing, storage and dispatching prescriptions in a network of hospitals. In this paper we have used the concept of resilience in supply chains to model the fact that healthcare networks cannot allow the presence of risk in the distribution of medicines and prescriptions. Also, we have used the concept of p-robustness to model the uncertainty in demand that is a natural process in healthcare because of the growing of population. A real life instance is solved, allowing to highlight the benefit of the p-robustness model compared to the deterministic one. This paper contributes to the healthcare location-allocation literature by addressing a real application of automation including the uncertainty in demand and considering specific features of the application relevant in real-world situations.

\section{ACKNOWLEDGMENT}

We thank pharmacists of Hopital Nord Ouest that gave us support in this research. Also we thank the bachelor student Aurélie Mélinand who was involved in the project. The first author would like to thank the Universidad del Rosario for their assistance in providing financial support in the research project, to the Universidad de la Sabana for providing partial financial support in his Ph.D program and also to Mines SaintÉtienne for hosting him during the research project. 


\section{REFERENCES}

[1] M. A. Ahadani, L. C. De Silva, I. Petra, M. F. A. Hameed, and T. S. Wong, "Low cost robotic medicine dispenser," Procedia Eng., vol. 41, no. Iris, pp. 202-209, 2012.

[2] S. M. Altawalbeh, M. I. Saul, A. L. Seybert, J. M. Thorpe, and S. L. Kane-Gill, "Intensive care unit drug costs in the context of total hospital drug expenditures with suggestions for targeted cost containment efforts," J. Crit. Care, vol. 44, pp. 77-81, 2018.

[3] J. Spinks, J. Jackson, C. M. Kirkpatrick, and A. J. Wheeler, "Disruptive innovation in community pharmacy - Impact of automation on the pharmacist workforce," Res. Soc. Adm. Pharm., vol. 13, no. 2, pp. 394-397, 2017.

[4] A. Murat, V. Verter, and G. Laporte, "A multi-dimensional shooting algorithm for the two-facility location-allocation problem with dense demand," Comput. Oper. Res., vol. 38, no. 2, pp. 450-463, Feb. 2011.

[5] M. Karatas and E. Yakıcı, "An iterative solution approach to a multiobjective facility location problem," Appl. Soft Comput., vol. 62, pp. 272-287, Jan. 2018.

[6] C. Ortiz-Astorquiza, I. Contreras, and G. Laporte, "Multi-level facility location problems,” Eur. J. Oper. Res., vol. In Press, p. In Press, Oct. 2017.

[7] F. Guerriero, G. Miglionico, and F. Olivito, "Location and reorganization problems: The Calabrian health care system case," Eur. J. Oper. Res., vol. 250, no. 3, pp. 939-954, 2016.

[8] S. S. R. Shariff, N. H. Moin, and M. Omar, "Location allocation modeling for healthcare facility planning in Malaysia," Comput. Ind. Eng., vol. 62, no. 4, pp. 1000-1010, May 2012.

[9] A. M. Mestre, M. D. Oliveira, and A. P. Barbosa-Póvoa, "Locationallocation approaches for hospital network planning under uncertainty,” Eur. J. Oper. Res., vol. 240, no. 3, pp. 791-806, 2014.

[10] F. Karamyar, J. Sadeghi, and M. M. Yazdi, "A Benders decomposition for the location-allocation and scheduling model in a healthcare system regarding robust optimization," Neural Comput. Appl., pp. 1-14, 2016.

[11] W. Zhang, K. Cao, S. Liu, and B. Huang, "A multi-objective optimization approach for healthcare facility location-allocation problems in highly developed cities such as Hong Kong," Comput. Environ. Urban Syst., vol. 59, pp. 220-230, 2016.

[12] C. Stummer, K. Doerner, A. Focke, and K. Heidenberger, "Determining location and size of medical departments in a hospital network: A multiobjective decision support approach," Health Care Manag. Sci., vol. 7, no. 1, pp. 63-71, 2004.

[13] N. Coskun and R. Erol, "An optimization model for locating and sizing emergency medical service stations," J. Med. Syst., vol. 34, no. 1, pp. 43-49, 2010.

[14] M. haji abbas and S. J. Hosseininezhad, "A robust approach to multi period covering location-allocation problem in pharmaceutical supply chain,” J. Ind. Syst. Eng., vol. 9, pp. 71-84, May 2016.

[15] F. Pietz, B. Benecke, E. K. Lee, and C. Hung Chen, "Modeling and optimizing the public-health infrastructure for emergency response," Interfaces (Providence)., vol. 39, no. 5, pp. 476-490, 2009.

[16] C. Boonmee, M. Arimura, and T. Asada, "Facility location optimization model for emergency humanitarian logistics," Int. J. Disaster Risk Reduct., vol. 24, pp. 485-498, Sep. 2017.

[17] J. Bowers, G. Mould, and C. Marshall, "Location of services and the impact on healthcare quality: Insights from a simulation of a musculoskeletal physiotherapy service,” J. Oper. Res. Soc., vol. 66, no. 7, pp. 1212-1221, 2015.

[18] A. Ahmadi-Javid, P. Seyedi, and S. S. Syam, "A survey of healthcare facility location," Comput. Oper. Res., vol. 79, pp. 223-263, Mar. 2017.

[19] E. D. Güne and S. Nickel, "Location Problems in Healthcare," in Location Science, 2015, pp. 555-579.

[20] A. Chauhan and A. Singh, "A hybrid multi-criteria decision making method approach for selecting a sustainable location of healthcare waste disposal facility," J. Clean. Prod., vol. 139, pp. 1001-1010, Dec. 2016.

[21] A. Nedjati, G. Izbirak, and J. Arkat, "Bi-objective covering tour location routing problem with replenishment at intermediate depots: Formulation and meta-heuristics," Comput. Ind. Eng., vol. 110, pp. 191-206, 2017.

[22] J. Meissner and O. V. Senicheva, "Approximate dynamic programming for lateral transshipment problems in multi-location inventory systems,” Eur. J. Oper. Res., vol. 265, no. 1, pp. 49-64, 2018.

[23] K. Gokbayrak and A. S. Kocaman, "A distance-limited continuous location-allocation problem for spatial planning of decentralized systems," Comput. Oper. Res., vol. 88, pp. 15-29, Dec. 2017.

[24] D. G. Mogale, M. Kumar, S. K. Kumar, and M. K. Tiwari, "Grain silo location-allocation problem with dwell time for optimization of food grain supply chain network," Transp. Res. Part E Logist. Transp. Rev., vol. 111, pp. 40-69, Mar. 2018.

[25] A. M. Saghiri and M. R. Meybodi, "Open asynchronous dynamic cellular learning automata and its application to allocation hub location problem," Knowledge-Based Syst., vol. 139, pp. 149-169, Jan. 2018.

[26] N. Ghaffarinasab, A. Motallebzadeh, Y. Jabarzadeh, and B. Y. Kara, "Efficient simulated annealing based solution approaches to the competitive single and multiple allocation hub location problems," Comput. Oper. Res., vol. 90, pp. 173-192, Feb. 2018.

[27] Y. Sheffi, The resilient enterprise : overcoming vulnerability for competitive advantage. MIT Press, 2005.

[28] C. Nemeth, R. Wears, D. Woods, E. Hollnagel, and R. Cook, Minding the Gaps: Creating Resilience in Health Care. Agency for Healthcare Research and Quality (US), 2008.

[29] P. Mensah and Y. Merkuryev, "Developing a resilient supply chain," Procedia - Soc. Behav. Sci., vol. 110, pp. 309-319, 2014.

[30] L. V. Snyder and M. S. Daskin, "Stochastic p -robust location problems," IIE Trans., vol. 38, no. 11, pp. 971-985, Nov. 2006. 